\title{
Retrospective Study of Acute Theophylline Intoxicated Cases Admitted to Poison Control Center, Ain-Shams University Hospitals from January 2007 to December 2008
}

\author{
Saad Naguib, Amany El Sayed, Hend El Helaly, and Sara Said ${ }^{1}$
}

${ }^{1}$ Forensic Medicine and Clinical Toxicology Department, Faculty of Medicine, Ain-Shams University, Cairo, Egypt

\begin{abstract}
Methylxanthines especially theophylline have been recognized as potent bronchodilators for of acute asthma over 65 years. Theophylline affects the cardiovascular (CV), neurological, gastrointestinal GI, and metabolic systems. One-hundred and ten patient's records with a discharge diagnosis of acute theophylline intoxication during the period from January 2007 to December 2008 at Poison control Center, Ain Shams University Hospitals were recruited. These patient's records were reported for history, clinical manifestations, investigations and management. It was noticeable that, majority of cases were Females between 10 to less than 30 years old. Gastrointestinal symptoms especially nausea and vomiting were the most frequent. Sinus tachycardia was present in $(85.5 \%)$ of acute overdose cases, while hypotension was found in only one case. Agitation was present in $(7.6 \%)$ of with single overdose, while $(1.92 \%)$ of those cases presented with hallucination. Only one case with single overdose presented with seizures. All patients were having toxic serum theophylline levels and hypokalemia. This work concluded that agitation, sinus tachycardia and hypokalemia were considered as prognostic factors. Moreover, the recorded clinical and laboratory parameters categorized all patients in to mild, moderate and severe cases. In-addition, this study recommended that prognostic factors reported as well as grading system outlined in this work must be assessed routinely and as early as possible to evaluate and improve the outcome the case.
\end{abstract}

\section{Introduction}

$\mathrm{T}$ Theophylline is an alkaloid closely related to caffeine and is available in plants (seeds of the Coffee plant, Coffee arabica, Thea sinensis) widely distributed geographically (Scheindlin, 2007).Acute toxicity may occur after single ingestion of more than $10 \mathrm{mg} \mathrm{kg}$ of theophylline while, acute on therapeutic intoxication occurs when ingestion of a toxic dose of theophylline in patients chronically using theophylline (Henry and Minton, 2011). Chronic intoxication occurs in patients who have been receiving repeated excessive doses of theophylline therapy for at least 72 hours. It may also result from impaired drug metabolism due to addition of a cytochrome P 450 inhibitor to a drug regimen or undercurrent illness (Shannon and Perry, 2005). The most significant morbidity and mortality of theophylline toxicity in acute overdose are secondary to cardiovascular and CNS effects i.e. Life threatening tachydysrhythmias and hypotension as well as refractory seizures can occur (Reilly, 2008).
This study aimed to specify both risk and prognostic factors of acute theophylline toxicity. In addition, it provided a tool of grading acute theophylline toxicity cases which may help in improving the course of management and better outcome.

\section{Subjects and methods}

The medical records were obtained and studied for 110 patients with a discharge diagnosis of acute theophylline intoxication and anonymous recording was done as well as all confidentiality issues were preserved. This work is a retrospective study that was carried out at Poison Control Center, Ain Shams University Hospitals (PCC, ASU) during the period from January 2007 to December 2008. An approval was taken from Professor Dr. Mohey El-Masry the director of the PCC. For all these cases; history, clinical manifestations, investigations and management were studied and analyzed. Patients were categorized as having single overdose and acute on 
chronic overdose. The study was investigating cases of all age group both male and females. The following parameters were reviewed:

1. The history of present illness (theophylline toxicity) was evaluated.

2. The clinical signs on presentation especially vital signs (pulse, blood pressure, temperature, respiratory rate), gastrointestinal (nausea, vomiting and diarrhea) neurological (agitation, hallucination and seizures) and cardiac manifestations (hypotension and arrhythmias).

3. Laboratory parameters included; serum theophylline, $\mathrm{K}+$ and random blood glucose levels as well as ECG recording.

4. The obtained data were expressed as numbers, percent, mean and standard deviation using student t-test, chisquare, Fisher's Exact Test and Linear Correlation Coefficient by SPSS V17.

\section{Results}

Table (1) showed incidence of gastrointestinal manifestations as regard type of toxicity. It was noticed that nausea and vomiting occurred in $(91.34 \%)$ of cases with single theophylline overdose and $(100 \%)$ of cases with acute on chronic overdose. Diarrhea occurred in $(4.8 \%)$ of cases with single overdose, while hematemesis occurred in $(6.73 \%)$ of those cases. (16.6\%) of cases with acute on chronic overdose presented with hematemesis. There was no significant difference between cases with single overdose and those with acute on chronic overdose as regard gastrointestinal manifestations as shown by Fisher's Exact Test.

Table (2),showed incidence of neurological manifestations as regard type of toxicity, (7.6\%) of cases with singe overdose presented with agitation, while $(1.92 \%)$ of cases were presented with hallucination. Only one case with single overdose presented with seizures. There was no significant difference between cases with single overdose and those with acute on chronic overdose regarding neurological manifestations as shown

Table (3), showed Fisher's Exact Test of cardiovascular manifestations, hypotension and sinus bradycardia were noticed in $(0.96 \%)$ of cases with single overdose. Sinus tachycardia occurred in $(85.5 \%)$ of cases with single overdose, while $(83.3 \%)$ presented of cases with acute on chronic overdoses. There was no significant difference between cases with single overdose and those with acute on chronic overdose as regard cardiovascular manifestations.

Incidence of theophylline level was shown in Table (4), majority of studied cases $(65.38 \%)$ with single overdose and $(66.66 \%)$ of those with acute on chronic overdose) were found to have serum theophylline level from $20-40 \mu \mathrm{g} / \mathrm{ml}$. serum theophylline levels in $(32.69 \%)$ of cases with single overdose and $(33.33 \%)$ of those with acute on chronic overdose were from $40-100 \mu \mathrm{g} / \mathrm{ml}$. Only $(1.92 \%)$ of cases with single overdose had serum theophylline levels $>100 \mu \mathrm{g} / \mathrm{ml}$. There was no significant difference between serum theophylline levels in cases with single overdose and those with acute on chronic overdose as shown by Fisher's Exact Test.

Table (5), showed incidence of serum potassium levels as regard type of toxicity. Potassium levels were in normal range $(3.5-5.5 \mathrm{mEq} / \mathrm{L})$ in $(21.15 \%)$ of cases with single overdose and in $(33.3 \%)$ of those with acute on chronic overdose. Majority of studied cases $(78.8 \%)$ of cases with single overdose and (66.6\%) of those with acute on chronic overdose had serum potassium level $<3.5 \mathrm{mEq} / \mathrm{L}$. There was no significant difference between serum potassium levels in cases with single overdose and those with acute on chronic overdose as shown by Fisher's Exact Test

Moreover, Table (6), showed incidence of random blood glucose levels as regard type of toxicity. Glucose levels in $(74 \%)$ of cases with single overdose and $(50 \%)$ of those with acute on chronic overdose were from $70-140 \mathrm{mg} / \mathrm{dl}$. (26\%) of cases with single overdose and $(50 \%)$ of those with acute on chronic overdose had glucose levels $>140 \mathrm{mg} / \mathrm{dl}$. There was no significant difference between random blood glucose levels in cases with single overdose and those with acute on chronic overdose as shown by Fisher's Exact Test.

Table (7), showed student "t" test comparative analysis between cases with single overdose and those with acute on chronic overdose as regard serum theophylline, glucose and $\mathrm{k}$ levels. There was significant difference between single overdose and acute on chronic overdose cases as regard $\mathrm{k}$ levels with no significant difference regarding other studied laboratory parameters.

In-addition, Table (8), showed incidence of duration of hospital stay of acute theophylline intoxicated cases. Majority of cases $(60.5 \%)$ of cases with single overdose and $(33.3 \%)$ of those with acute on chronic overdose, stayed in the hospital for one day. Nearly, $(33.6 \%)$ of cases with single overdose and $(33.3 \%)$ of those with acute on chronic overdose stayed for 2 days. $(1.92 \%)$ of cases with singe overdose and $16.6 \%$ of those with acute on chronic overdose stayed for 3 days and the same percent of cases stayed for 4 days. No significant difference was found between single overdose and acute on chronic overdose cases as regard duration of hospital stay as shown by Fisher's Exact Test.

Table (9), showed incidence of site of admission of theophylline intoxicated cases. Majority of patients $(94.23 \%)$ of cases with single overdose and $(83.3 \%)$ of those with acute on chronic overdose were admitted to in-patient, while only $(5.77 \%)$ of cases with single overdose and (16.6\%) of those with acute on chronic overdose were admitted in the intensive care unit (I C U). No significant difference was found between single 
overdoses and acute on chronic overdose cases as regard site of admission as shown by Fisher's Exact Test.

Incidence of different lines of management applied to theophylline intoxicated cases were shown in Table (10). According to time of presentation, mode of poisoning, clinical status of the patient, the following lines of management were reported to be supplied as shown in the table; emesis was used in $(66.35 \%)$ of single overdose. All cases (100\%) received repeated doses of activated charcoal. (8.65\%) of cases who suffered agitation and seizures were treated with sedatives hypnotics (Valium) while antiepileptic (Epanutin) was used in only $(0.96 \%)$ of cases. Hematemsis in $(6.73 \%)$ of cases with acute overdose as well as (16.67) acute on chronic overdose were treated by (Dicinon, Cyclocapron and Konakion). Intravenous fluids (saline) were used to resuscitate one patient who was presented with hypotension and no vasopressors were used. Antiemetics as (Primpran) was used for almost all cases and $\mathrm{H} 2$ receptors blocker (Zantac) were used in $(92.31 \%)$ of acute single overdose cases and almost all those presented with acute on chronic overdose who suffered vomiting. Potassium supplementations were used in $(34.62 \%)$ of acute single overdose cases. Only two acute single overdose cases required hemodialysis. No significant difference was found between single overdoses and acute on chronic overdose cases as regard different lines of treatment as shown by Fisher's Exact Test.

\section{Prognostic Factors}

Correlations between serum theophylline level and clinical parameters as regard different type of toxicity were shown in Table (11). There was significant difference in serum theophylline level in cases with agitation and tachycardia when compared with cases without such manifestations. No significant difference was noticed in serum theophylline level between both types of cases regarding the other clinical parameters

Moreover, Correlations between theophylline level and studied Biochemical parameters as regard different type of toxicity were shown in Table (12) and Figures $(1,2, \& 3)$.It was noticed that there was positive correlation between serum theophylline and random serum glucose levels in cases with single overdose, while no correlation was found between serum theophylline and random serum glucose levels in those with acute on chronic overdose. However, there was significant negative correlation between serum theophylline and potassium levels in cases with single overdose and those with acute on chronic overdose.

In-addition, correlations between serum theophylline level and ingested theophylline dose as regard different type of theophylline toxicity were shown in Table (13) and Figure (4). There was significant correlation between plasma theophylline level and ingested dose in cases with single overdose, while no correlation was found between serum theophylline levels and ingested dose in cases with acute on chronic overdose. However, correlation between serum theophylline level and ECG findings were shown in Table (14), revealed that there was no correlation between serum theophylline level and PR interval and QRS complex duration in the studied cases.

Correlation between stay factor (delay time $x$ ingested theophylline dose) and hospital stay was shown in Table (15). There was no correlation between stay factor and hospital stay. As there was no uniformed protocol for discharge of patients from the PCC, ASU, so this result may not be a reliable result.

\section{Grading of patients}

Table (16) showed Chi-square statistical analysis of clinical parameters and laboratory parameters in mild, moderate and severe theophylline toxicity according to theophylline level. Caravati (2004) who mentioned that mild theophylline intoxication occurs between serum theophylline levels 20 and $40 \mathrm{mcg} \backslash \mathrm{ml}$, moderate toxicity at level $40-100 \mathrm{mcg} \backslash \mathrm{ml}$ and severe toxicity at more than $100 \mathrm{mcg} \backslash \mathrm{ml}$.

As regard mild cases pulse rate was from less than $100-120 \mathrm{bpm}$. in (62\%) of those cases and (13\%) had 120-140bpm. While systolic blood pressure was from $100-120 \mathrm{mmHg}$ in $(65 \%)$ of mild cases and $>120 \mathrm{mmHg}$ in $(34 \%)$ of those cases. Potassium level was in normal range $(3.5-5.5 \mathrm{mEq} / \mathrm{L})$ in $(23 \%)$ of mild cases, while $(47 \%)$ of those cases had potassium levels $(3-3.5 \mathrm{mEq} / \mathrm{L})$ and $(27 \%)$ had $(2.5-3 \mathrm{mEq} / \mathrm{L})$. Random blood glucose level was from $70-140 \mathrm{mg} / \mathrm{dl}$ in (76.2\%) of mild cases.

According to this table, moderate theophylline toxicity cases had; pulse rate from $100-120 \mathrm{bpm}$ in $(36 \%)$ of cases and 120-140 bpm in (58\%) of, while systolic blood pressure was $100-120 \mathrm{mmHg}$ in $(88 \%)$ of those cases. Potassium level in

(19.4) was within normal range (3.5-5.5 $\mathrm{mEq} / \mathrm{L}$.) while, $(69.4 \%)$ of those cases had potassium level from $(2.5$ to $3 \mathrm{mEq} / \mathrm{L})$. Random blood glucose level was from $(70-140 \mathrm{mg} / \mathrm{dl})$ in $(69.4 \%)$ of moderate cases; while $(30.5 \%)$ of those cases had random blood glucose level from (140 to $200 \mathrm{mg} / \mathrm{dl})$.

In-addition, pulse rate was $\geq 140 \mathrm{bpm}$ in $(100 \%)$ of severe cases, while systolic blood pressure was < $100 \mathrm{mmHg}$ in $(100 \%)$ of those cases. Potassium level was from ( 2 to $5.3 \mathrm{mEq} / \mathrm{L})$ in $(100 \%)$ of cases. Random blood glucose level was from (140 to $200 \mathrm{mg} / \mathrm{dl})$ in $(50 \%)$ of severe cases, while $(50 \%)$ of those cases had random blood glucose level ( $\geq 200 \mathrm{mg} / \mathrm{dl}$ ). There was significant difference between mild, moderate and severe cases as regard pulse rate and systolic blood pressure. While there was no significant difference was detected between mild, moderate and severe cases as regard other clinical manifestations; gastrointestinal (nausea, vomiting and diarrhea) and neurological manifestations (agitation, hallucination and seizures) as shown by this table. Also, there was significant difference between mild, moderate 
and severe cases as regard serum potassium and random blood glucose levels.

Table (1): Statistical analysis (Fisher's Exact Test) of gastrointestinal manifestations as regard type of toxicity

\begin{tabular}{|l|c|c|c|c|c}
\hline \multirow{2}{*}{ GIT manifestations } & \multicolumn{2}{|c|}{ Single overdose (N=104) } & Acute on chronic overdose (N=6) & \multirow{2}{*}{ P-value } \\
\cline { 2 - 5 } & $\mathrm{N}$ & $\mathbf{\%}$ & $\mathrm{N}$ & $\%$ & 100 \\
\hline Nausea and vomiting & 95 & 91.34 & 6 & 0 & 0.592 \\
\hline Diarrhea & 5 & 4.8 & 0 & 16.6 & 0.751 \\
\hline Hematemesis & 7 & 6.73 & 1 & 0 & 0.371 \\
\hline None & 9 & 8.6 & 0 & 6 & 0.592 \\
\hline Total & & 104 & & 6 \\
\hline
\end{tabular}

$* P<0.05=$ significant, $P \geq 0.05=$ insignificant

Table (2): Statistical analysis (Fisher's Exact Test) of neurological manifestations as regard type of toxicity

\begin{tabular}{|l|c|c|c|c|c}
\hline \multirow{2}{*}{ CNS manifestations } & \multicolumn{2}{|c|}{ Single overdose (N=104) } & \multicolumn{2}{c|}{ Acute on chronic overdose (N=6) } & \multirow{2}{*}{ P-value } \\
\cline { 2 - 5 } & $\mathbf{N}$ & $\mathbf{\%}$ & $\mathbf{N}$ & $\mathbf{\%}$ & \\
\hline Agitation & 8 & 7.6 & 0 & 0 & 0.629 \\
\hline Seizures & 1 & 0.96 & 0 & 0 & 0.945 \\
\hline Hallucination & 2 & 1.92 & 0 & 0 & 0.893 \\
\hline None & 93 & 89.4 & 6 & 100 & 0.523 \\
\hline Total & 104 & & 6 & & \\
\hline
\end{tabular}

$* P<0.05=$ significant, $P \geq 0.05=$ insignificant

Table (3): Statistical analysis (Fisher's Exact Test) of cardiovascular manifestations as regard type of toxicity

\begin{tabular}{|l|c|c|c|c|c}
\hline \multirow{2}{*}{ CVS manifestations } & \multicolumn{2}{|c|}{ Single overdose (N=104) } & Acute on chronic overdose (N=6) & \multirow{2}{*}{ P- value } \\
\cline { 2 - 5 } & $\mathrm{N}$ & $\mathbf{\%}$ & $\mathrm{N}$ & $\%$ & \\
\hline Sinus tachycardia & 89 & 85.5 & 5 & 83.3 & 0.945 \\
\hline Sinus bradycardia & 1 & 0.96 & 0 & 0 & 0.62 \\
\hline Hypotension & 1 & 0.96 & 0 & 0 & 0.62 \\
\hline None & 14 & 12.7 & 1 & 16.6 & 0.945 \\
\hline Total & 104 & & 6 & & \\
\hline
\end{tabular}

$* P<0.05=$ significant, $P \geq 0.05=$ non-significant

Table (4): Statistical Analysis (Fisher's Exact Test) of theophylline level

\begin{tabular}{|l|c|c|c|c|c|}
\hline \multirow{2}{*}{ Theophylline level $(\boldsymbol{\mu g} / \mathbf{m L})$} & \multicolumn{2}{|c|}{ Single overdose $(\mathbf{N}=\mathbf{1 0 4})$} & \multicolumn{2}{|c|}{ Acute on chronic overdose (N=6) } & \multirow{2}{*}{ P- value } \\
\cline { 2 - 6 } & $\mathbf{N}$ & $\mathbf{\%}$ & $\mathbf{N}$ & $\mathbf{\%}$ & 0.659 \\
\hline $20-40$ & 68 & 65.38 & 4 & 66.66 & 0.643 \\
\hline$>40-100$ & 34 & 32.69 & 2 & 33.33 & 0.893 \\
\hline$>100$ & 2 & 1.92 & 0 & 0 & \\
\hline Total & 104 & & 6 & & \\
\hline
\end{tabular}

$* P<0.05=$ significant, $P \geq 0.05=$ non-significant

Table (5): Statistical Analysis (Fisher's Exact Test) of potassium level

\begin{tabular}{|l|c|c|c|c|c}
\hline \multirow{2}{*}{ Potassium level (mEq/L) } & \multicolumn{2}{|c|}{ Single overdose $\mathbf{( N = 1 0 4 )}$} & \multicolumn{2}{|c|}{ Acute on chronic overdose (N=6) } & \multirow{2}{*}{ P value } \\
\cline { 2 - 6 } & $\mathbf{N}$ & $\mathbf{\%}$ & $\mathbf{N}$ & $\mathbf{\%}$ & 0.39 \\
\hline$<3.5$ & 82 & 78.8 & 4 & 66.6 & 0.39 \\
\hline $3.5-5.5$ & 22 & 21.15 & 2 & 33.3 & \\
\hline$>5.5$ & 0 & 0 & 0 & 0 & \\
\hline Total & 104 & & 6 & & \\
\hline
\end{tabular}

$* P<0.05=$ significant,$P \geq 0.05=$ non-significant

Table (6): Statistical Analysis (Fisher's Exact Test)of random blood glucose level

\begin{tabular}{|l|c|c|c|c|c}
\hline \multirow{2}{*}{ Glucose level (mg/dL) } & \multicolumn{2}{|c|}{ Single overdose (N=104) } & \multicolumn{2}{|c|}{ Acute on chronic overdose (N=6) } & \multirow{2}{*}{ P value } \\
\cline { 2 - 6 } & $\mathbf{N}$ & $\mathbf{\%}$ & $\mathbf{N}$ & $\mathbf{\%}$ & \\
\hline$<70$ & 0 & 0 & 0 & 0 & 0 \\
\hline $70-140$ & 77 & 74 & 3 & 50 & 0.202 \\
\hline$>140$ & 27 & 26 & 3 & 50 & 0.202 \\
\hline Total & 104 & & 6 & & \\
\hline
\end{tabular}

$* P<0.05=$ significant, $P \geq 0.05=$ non-significant 
Table (7): Student's T-test statistical analysis of theophylline level, potassium and random blood glucose as regard type of theophylline toxicity

\begin{tabular}{|c|c|c|c|c|c|c|}
\hline \multirow{2}{*}{ Parameter } & \multicolumn{2}{|c|}{ Single overdose $(\mathbf{N}=\mathbf{1 0 4})$} & \multicolumn{2}{c|}{ Acute on chronic overdose (N=6) } & \multicolumn{2}{c|}{ T-test } \\
\cline { 2 - 7 } & Mean & \pm SD & Mean & \pm SD & t & P-value \\
\hline Theophylline level $(\mu \mathrm{g} / \mathrm{mL})$ & 49.309 & 20.424 & 47.333 & 19.044 & 0.246 & 0.814 \\
\hline Potassium level $(\mathrm{mEq} / \mathrm{L})$ & 3.044 & 0.403 & 3.483 & 0.962 & -2.352 & $0.020^{*}$ \\
\hline Blood glucose $(\mathrm{mg} / \mathrm{dL})$ & 128.837 & 48.991 & 133.500 & 33.483 & -0.230 & 0.819 \\
\hline
\end{tabular}

$* P<0.05=$ significant, $P \geq 0.05=$ non-significant

Table (8): Statistical Analysis (Fisher's Exact Test) of duration of hospital stay

\begin{tabular}{|l|c|c|c|c|c|}
\hline \multirow{2}{*}{ Hospital stay (days) } & \multicolumn{2}{|c|}{ Single overdose (N=104) } & \multicolumn{2}{c|}{ Acute on chronic overdose (N=6) } & \multirow{2}{*}{ P-value } \\
\cline { 2 - 5 } & $\mathbf{N}$ & $\mathbf{\%}$ & $\mathbf{N}$ & $\mathbf{\%}$ & \\
\hline 1 & 63 & 60.5 & 2 & 33.3 & 0.186 \\
\hline 2 & 35 & 33.6 & 2 & 33.3 & 0.677 \\
\hline 3 & 2 & 1.92 & 1 & 16.6 & 0.156 \\
\hline 4 & 2 & 1.92 & 1 & 16.6 & 0.156 \\
\hline 5 & 1 & 0.96 & 0 & 0 & 0.945 \\
\hline 6 & 1 & 0.96 & 0 & 0 & 0.945 \\
\hline Total & 104 & & 6 & 100.00 & \\
\hline
\end{tabular}

$* P<0.05=$ significant, $P \geq 0.05=$ non-significant

Table (9): Statistical Analysis (Fisher's Exact Test) of incidence of site of admission

\begin{tabular}{|l|c|c|c|c|c|}
\hline \multirow{2}{*}{ Site of admission } & \multicolumn{2}{|c|}{ Single overdose $\mathbf{( N = 1 0 4 )}$} & \multicolumn{2}{c|}{ Acute on chronic overdose (N=6) } & \multirow{2}{*}{ P -value } \\
\cline { 2 - 5 } & $\mathbf{N}$ & $\mathbf{\%}$ & $\mathbf{N}$ & $\mathbf{\%}$ & \\
\hline In patient & 98 & 94.23 & 5 & 83.3 & 0.364 \\
\hline ICU & 6 & 5.77 & 1 & 16.6 & 0.364 \\
\hline Total & 104 & 100.00 & 6 & 100.00 & \\
\hline
\end{tabular}

$* P<0.05=$ significant, $P \geq 0.05=$ non-significant

Table (10): Statistical analysis (Fisher's Exact Test) of different measures of treatment applied intoxicated cases

\begin{tabular}{|c|c|c|c|c|c|}
\hline \multirow{2}{*}{ Measure } & \multicolumn{2}{|c|}{ Single overdose $(\mathrm{N}=104)$} & \multicolumn{2}{|c|}{ Acute on chronic overdose $(\mathrm{N}=6)$} & \multirow{2}{*}{ P -value } \\
\hline & $\mathbf{N}$ & $\%$ & $\mathbf{N}$ & $\%$ & \\
\hline \multicolumn{6}{|l|}{ Emergency treatment } \\
\hline Sedatives hypnotics & 9 & 8.65 & 0 & 0.00 & 0.592 \\
\hline Antiepileptics & 1 & 0.96 & 0 & 0.00 & 0.945 \\
\hline Treatment of hematemesis & 7 & 6.73 & 1 & 16.67 & 0.371 \\
\hline \multicolumn{6}{|l|}{ Supportive treatment } \\
\hline I.V fluids & 104 & 100.00 & 6 & 100.00 & 0.893 \\
\hline Antiemetics & 102 & 98.08 & 6 & 100.00 & 0.893 \\
\hline H2 receptors blockers & 96 & 92.31 & 6 & 100.00 & 0.629 \\
\hline Potassium supplementations & 36 & 34.62 & 0 & 0.00 & 0.086 \\
\hline \multicolumn{6}{|l|}{ Decontamination } \\
\hline Emesis & 69 & 66.35 & 4 & 66.67 & 0.677 \\
\hline Charcoal & 104 & 100.00 & 6 & 100.00 & 0.893 \\
\hline \multicolumn{6}{|l|}{ Enhancement } \\
\hline MDAC & 104 & 100.00 & 6 & 100.00 & 0.895 \\
\hline Hemodialysis & 2 & 1.88 & 0 & 0.00 & 0.844 \\
\hline
\end{tabular}

$* P<0.05=$ significant, $P \geq 0.05=$ non-significant 
Table (11): Student t-test analysis of serum theophylline level with some clinical parameters

\begin{tabular}{|l|r|l|c|l|c|c|}
\hline \multirow{2}{*}{} & \multicolumn{7}{|c|}{ Theophylline level } \\
\cline { 2 - 7 } & \multicolumn{2}{|c|}{ Cases without } & \multicolumn{2}{c|}{ Cases with } & \multicolumn{2}{c|}{ T-test } \\
\cline { 2 - 7 } & Mean & \pm SD & Mean & \pm SD & t & P-value \\
\hline Nausea and vomiting & 47.4667 & 19.8236 & 40.121 & 21.325 & -1.076 & 0.284 \\
\hline Hematemsis & 38.501 & 20.7508 & 36.86 & 15.53 & 0.19 & 0.85 \\
\hline Hallucination & 39.4776 & 20.2762 & 29.33 & 21.46 & 0.77 & 0.45 \\
\hline Agitation & 36.605 & 15.2936 & 68.33 & 40.45 & 5.1 & $<0.001^{*}$ \\
\hline Tachycardia & 39.3028 & 20.4103 & 42.351 & 19.712 & -4.075 & $0.001^{*}$ \\
\hline
\end{tabular}

$* P<0.05=$ significant, $P \geq 0.05=$ non-significant

Table (12): Pearson correlation test between serum theophylline level and serum potassium and random blood glucose level as regard type of theophylline toxicity

\begin{tabular}{|c|l|c|c|}
\hline \multicolumn{4}{|c|}{ Theophylline level } \\
\hline Parameter & \multicolumn{1}{|c|}{ Type of toxicity } & r & P-value \\
\hline \multirow{2}{*}{ Serum potassium level } & Single overdose & -0.211 & $0.032^{*}$ \\
\cline { 2 - 4 } & Acute on chronic overdose & -0.928 & $0.008^{*}$ \\
\hline Random blood glucose & Single overdose & 0.362 & $0.000^{*}$ \\
\cline { 2 - 4 } & Acute on chronic overdose & 0.771 & 0.072 \\
\hline
\end{tabular}

$* P<0.05=$ significant, $P \geq 0.05=$ non-significant

Table (13): Pearson correlation test between serum theophylline level and theophylline dose as regard type of theophylline toxicity

\begin{tabular}{|l|l|c|c|}
\hline \multicolumn{2}{|c|}{ Theophylline level $(\boldsymbol{\mu g} / \mathbf{d L})$} & $\mathbf{r}$ & $\mathbf{p}$ \\
\hline \multirow{2}{*}{ Theophylline dose } & Single overdose & 0.327 & $0.000^{*}$ \\
\cline { 2 - 4 } & Acute on chronic overdose & 0.812 & 0.05 \\
\hline
\end{tabular}

$* P<0.05=$ significant, $P \geq 0.05=$ non-significant

Table (14): Pearson correlation test between serum theophylline level and studied ECG findings as regard type of theophylline toxicity

\begin{tabular}{|c|l|c|c|}
\hline \multicolumn{4}{|c|}{ theophylline level } \\
\hline \multirow{2}{*}{ ECG findings } & \multicolumn{1}{|c|}{ Type of toxicity } & r & P-value \\
\hline \multirow{2}{*}{ PR interval } & Single overdose & -0.182 & 0.064 \\
\cline { 2 - 4 } & Acute on chronic overdose & -0.393 & 0.441 \\
\hline QRS complex duration & Single overdose & -0.294 & 0.571 \\
\cline { 2 - 4 } & Acute on chronic overdose & -0.083 & 0.4 \\
\hline
\end{tabular}

$* P<0.05=$ significant, $P \geq 0.05=$ non-significant

Table (15): Pearson correlation between stay factor and hospital stay

\begin{tabular}{|c|c|c|}
\hline Stay factor & r & p-value \\
\hline Hospital stay & -0.098 & 0.309 \\
\hline
\end{tabular}

$* P<0.05=$ significant, $P \geq 0.05=$ non-significant 
Table (16): Chi-square statistical analysis of clinical parameters and laboratory parameters in mild, moderate and severe patients groups

\begin{tabular}{|c|c|c|c|c|c|c|c|c|}
\hline & \multicolumn{2}{|c|}{ Mild } & \multicolumn{2}{|c|}{ Moderate } & \multicolumn{2}{|c|}{ Severe } & \multicolumn{2}{|c|}{ Chi-square } \\
\hline & $\mathbf{N}$ & $\%$ & $\mathbf{N}$ & $\%$ & $\mathbf{N}$ & $\%$ & $\mathbf{X}^{2}$ & P-value \\
\hline \multicolumn{9}{|l|}{ GIT } \\
\hline Nausea & 65 & 90 & 34 & 94 & 2 & 100 & \multirow{4}{*}{9.835} & \multirow{4}{*}{0.132} \\
\hline Vomiting & 65 & 90 & 34 & 94 & 2 & 100 & & \\
\hline Diarrhea & 3 & 4.1 & 2 & 5 & 0 & 0 & & \\
\hline Hematemesis & 1 & 1.3 & 7 & 19 & 0 & 0 & & \\
\hline \multicolumn{9}{|l|}{ CVS } \\
\hline \multicolumn{9}{|l|}{ Pulse (bpm) } \\
\hline$<100$ & 16 & 22.22 & 0 & 0 & 0 & 0 & \multirow{4}{*}{71.834} & \multirow{4}{*}{$0.000^{*}$} \\
\hline $100-120$ & 45 & 62 & 13 & 36 & 0 & 0 & & \\
\hline $120-140$ & 10 & 13 & 21 & 58 & 0 & 0 & & \\
\hline$>140$ & 1 & 1.3 & 2 & 5 & 2 & 100 & & \\
\hline \multicolumn{9}{|c|}{ Systolic blood pressure (mmHg) } \\
\hline$<100$ & 0 & 0 & 4 & 11 & 2 & 100 & \multirow{3}{*}{55.335} & \multirow{3}{*}{$0.000 *$} \\
\hline $100-120$ & 47 & 65 & 32 & 88 & 0 & 0 & & \\
\hline$>120$ & 25 & 34 & 0 & & 0 & 0 & & \\
\hline \multicolumn{9}{|l|}{ CNS } \\
\hline Agitation & 1 & 1.3 & 6 & 16 & 2 & 100 & \multirow{3}{*}{5.159} & \multirow{3}{*}{0.271} \\
\hline Hallucination & 1 & 1.3 & 1 & 2.7 & 0 & 0 & & \\
\hline Seizures & 0 & 0 & 0 & 0 & 1 & 50 & & \\
\hline \multicolumn{9}{|c|}{ Lab parameters } \\
\hline \multicolumn{9}{|c|}{ Potassium $(\mathrm{mEq} / \mathrm{L})$} \\
\hline $3.5-5.5$ & 17 & 23 & 7 & 19.4 & 0 & 0 & \multirow{4}{*}{34.053} & \multirow{4}{*}{$0.000 *$} \\
\hline $3-3.5$ & 34 & 47 & 2 & 5 & 0 & 0 & & \\
\hline $2.5-3$ & 20 & 27 & 25 & 69.4 & 2 & 100 & & \\
\hline$<2.5$ & 1 & & 2 & 5.5 & 0 & 0 & & \\
\hline \multicolumn{9}{|c|}{ Glucose (mg/dL) } \\
\hline $70-140$ & 55 & 76.2 & 25 & 69.4 & 0 & 0 & \multirow{3}{*}{22.618} & \multirow{3}{*}{$0.000 *$} \\
\hline $140-200$ & 6 & 8 & 11 & 30.5 & 1 & 50 & & \\
\hline$>200$ & 1 & 1.3 & 0 & 0 & 1 & 50 & & \\
\hline
\end{tabular}

$* P<0.05=$ significant,$P \geq 0.05=$ non-significant

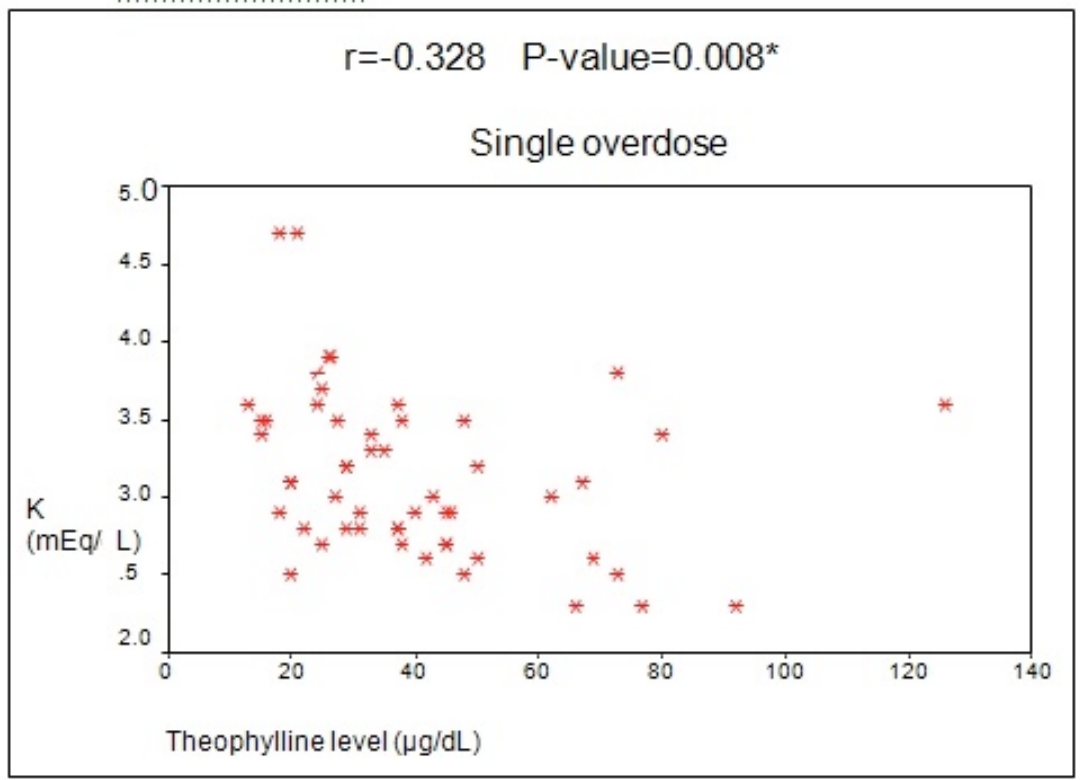

Figure (1): Correlation between serum theophylline serum and potassium levels in single overdose. 


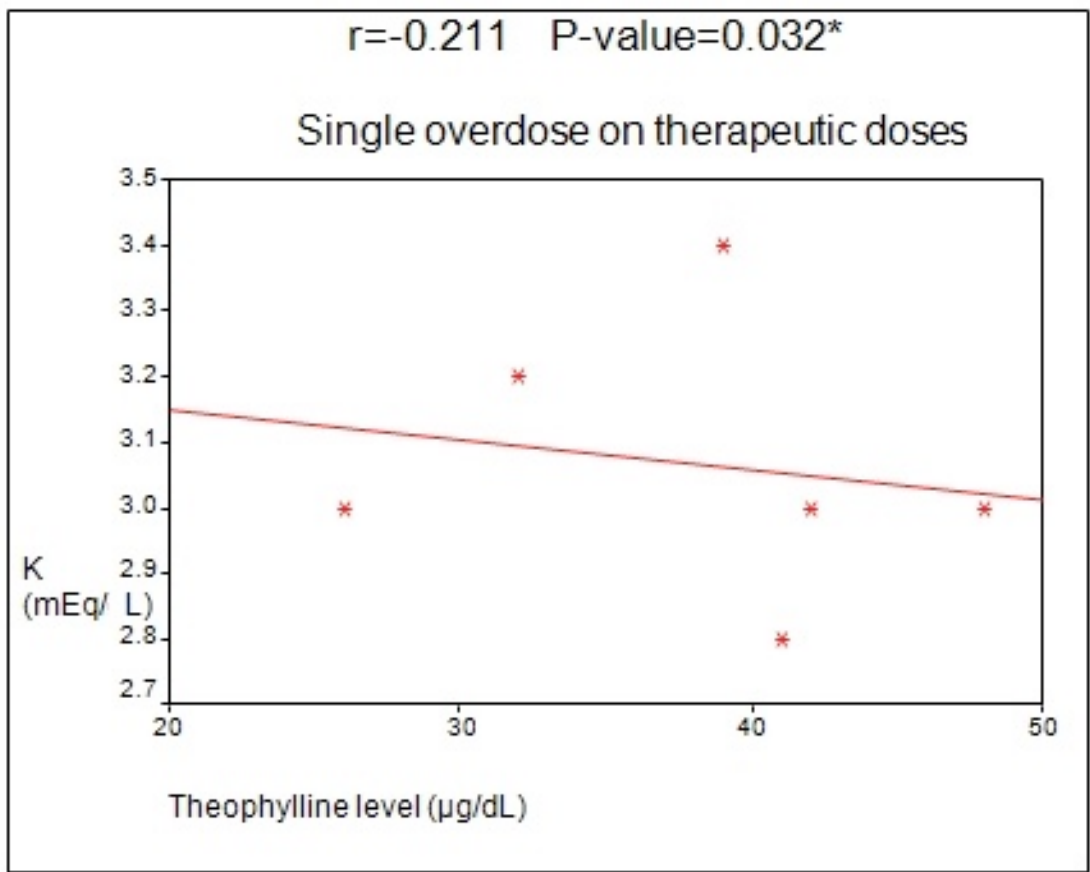

Figure (2): Correlation between serum theophylline and serum potassium levels in acute on chronic overdose.

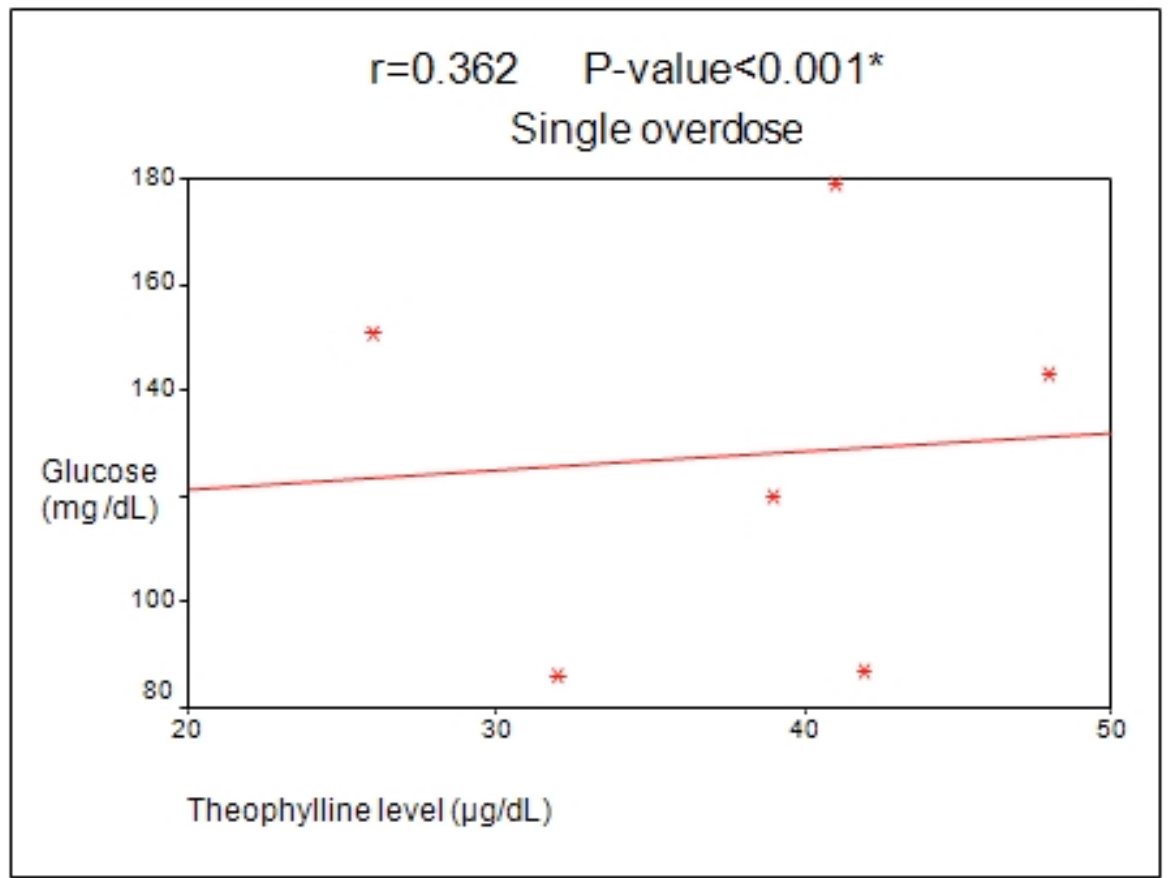

Figure (3): Correlation between serum theophylline and random blood glucose levels in single overdose. 


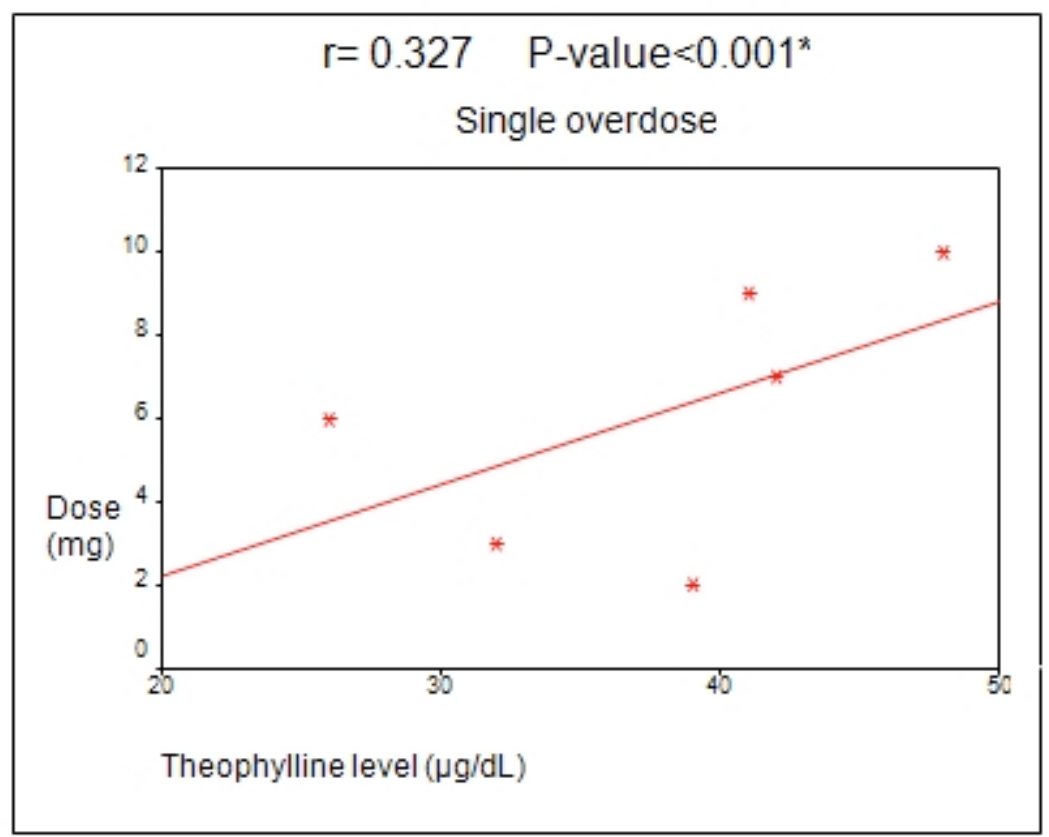

Figure (4): Correlation between serum theophylline level and theophylline dose in single overdose.

\section{Discussion}

Despite declining use of theophylline, it remains an important cause of intoxication with significant morbidity and mortality, due to theophylline's narrow therapeutic index (Raymond, 2010). This study is a retrospective study included 110 diagnosed theophylline toxicity that admitted to the Poison Control Center, Cairo, Ain Shams University hospitals (PCC, ASU.) during the period from January 2007 to December 2008.

The present study revealed that majority of cases presented with nausea and vomiting occurred with single overdose, and all those with acute on chronic overdose. Minority of cases presented with diarrhea and hematemesis. Perry, (2011) reported that nausea and vomiting occur in about $(97 \%)$ of patients with single theophylline overdose. Shannon and Perry (2005) attributed the gastrointestinal manifestations of theophylline toxicity to the central action of theophylline on the medulla as well as its local gastrointestinal actions.

Moreover, minority of acute single overdose cases presented with agitation, and hallucination with only one case with single overdose presented with seizures. No agitation, hallucination nor seizures were reported in patients with acute on chronic overdose. This result in agreement with Kato et al. (2007) reported that only $(4 \%)$ of theophylline intoxicated cases presented with seizures. Raymond, (2010), explained this seizure is due to adenosine receptors blockade in CNS.
In the present study, majority of cases with single overdose theophylline toxicity had arrhythmia, tachycardia. While, only one case suffered from hypotension with single theophylline overdose toxicity this goes with Rutten et al. (2005) who described a case of acute that was presented with severe hypotension. Inaddition, Kumar (2004) in the Christian Medical College, India reported that $100 \%$ of cases with acute single theophylline over dosage manifested with sinus tachycardia and he clarified that due to increased plasma catecholamines.

Laboratory parameters interpretations in this present study showed that, majority of studied cases were found to have serum theophylline level from (20$40 \mu \mathrm{g} / \mathrm{ml}$ ) with only one third of the cases had serum theophylline level from $40-100 \mu \mathrm{g} / \mathrm{ml}$. Only $(1.92 \%)$ of cases with single overdose had serum theophylline levels $>100 \mu \mathrm{g} / \mathrm{ml}$ with no significant differences between serum theophylline levels in patients with single overdose and serum theophylline levels in those with acute on chronic overdose. This result goes parallel with Shannon (1993) who mentioned that no differences in peak serum theophylline concentration between patients with single overdose, those with acute on chronic overdose and those with chronic theophylline toxicity.

No significant difference was found between incidences of serum potassium, random glucose levels as regard type of toxicity with majority of cases with hypokalemia. This result align with Hoffman (2006) who 
mentioned that hypokalemia occur in about $(85 \%)$ of patients with single overdose and he attributed that to catecholamines excess with intracellular movement of potassium. However, hyperglycemia occurred in (26.3\%) of cases with $(25 \%)$ of cases with single overdose, compared with $(50 \%)$ of those with acute on chronic theophylline overdose. This is in agreement with Tesfaye et al. (2008) who clarified theophylline induced hyperglycemia as a rise in plasma catecholamines.

Gut decontamination and supportive measures were done for the majority of cases. Eight patients, with hypokalemia, agitation, seizures, hypotension and hematemesis were admitted to ICU. One hundred and two patients were managed in the in-patient department showed complete recovery. Only one female died after development of cardiac arrest and she was the only case who presented with seizures, $(\mathrm{K}=2.3 \mathrm{meq} / \mathrm{l})$ and (random blood glucose $=255 \mathrm{mg} / \mathrm{dl}$ ). Only two patients required hemodialysis both of them had serum theophylline level $>90 \mu \mathrm{g} / \mathrm{ml}$ and manifested with agitation and hematemesis and they were completely recovered and discharged. Borkan (2002) suggested that hemodialysis should be performed prophylactically if serum theophylline level is $>80-100 \mu \mathrm{g} / \mathrm{ml}$ after an acute ingestion or Patients present with life-threatening intoxication such as seizure, hypotension or cardiac arrhythmias or patient unable to tolerate activated charcoal.

The present study showed that mean serum theophylline level was positively correlated with agitation and tachycardia, serum glucose level and ingested theophylline dose in acute single overdose. However, there was there was negative correlation between serum theophylline and serum potassium levels in patients with single overdose and those with acute on chronic overdose. In-addition, No correlation was found between serum theophylline and serum glucose level as well as ingested theophylline dose in patients with acute on chronic overdose. Moreover, there was no correlation between theophylline level and PR interval and QRS complex duration. These results go hand in hand with Shannon (1999) who mentioned that there was a significant negative correlation between serum theophylline and serum potassium levels in patients with single overdose and those with acute on chronic overdose. He also mentioned that there was a significant positive correlation between serum theophylline and serum glucose levels in patients with single overdose, while there was no correlation between serum theophylline and glucose levels in those with acute on chronic overdose. Moreover, Lowry et al. (2001), studied three cases of acute theophylline toxicity who exhibited agitation with serum theophylline level ranging from $(55 \mu \mathrm{g} / \mathrm{ml}$ to $123 \mu \mathrm{g} / \mathrm{ml})$. Perry (2011) added that cardiac arrhythmias was significantly associated with high serum theophylline levels in (119) cases with single overdose.

From these studied correlations it could be considered that presence of agitation; sinus tachycardia and hypokalemia were prognostic factors of both single and acute on chronic theophylline overdose, while hyperglycemia and ingested theophylline dose were prognostic factors of single theophylline overdose.

According to Caravati (2004) who mentioned that mild theophylline intoxication occurs between serum theophylline levels (20 to $40 \mu \mathrm{g} / \mathrm{ml}$ ), moderate toxicity at level $(40$ to $100 \mu \mathrm{g} / \mathrm{ml})$ and severe toxicity at more than $(100 \mu \mathrm{g} / \mathrm{ml})$.Based on these data, the present study reported that, there was significant difference between mild, moderate and severe cases as regard pulse rate and systolic blood pressure. No significant difference was found between mild, moderate and severe cases as regard other clinical manifestations. However, there was significant difference between mild, moderate and severe cases as regard serum potassium and random blood glucose levels.

These results go in agreement with Rahat et al. (2005) who mentioned that sinus tachycardia (pulse rate $\leq 120 \mathrm{bpm}$.) and normal blood pressure are common findings in mild theophylline toxicity cases. They also mentioned, that serum potassium level in mild theophylline toxicity ranged from $(2.5-3.5 \mathrm{mEq} / \mathrm{L})$, while serum potassium level in moderate theophylline toxicity was $\leq(2.5 \mathrm{mEq} / \mathrm{L})$. However, they mentioned that systolic arterial blood pressure in moderate theophylline toxicity is at least $(60 \mathrm{mmHg})$. Lim et al. (2005) mentioned that hypotension is a life threatening manifestation of severe theophylline toxicity.

\section{Conclusion}

The study revealed that majority of cases was females between 10 to less than 30 years old. Gastrointestinal symptoms especially nausea and vomiting were the most frequent presentations. Moreover, majority of cases had sinus tachycardia, while hypotension was found in only one case with single overdose theophylline toxicity. Agitation and hallucination were less frequent presentations with only one case with single overdose presented with seizures. In-addition, all patients were 
having toxic serum theophylline levels and hypokalemia. It could be considered that, presence of agitation, sinus tachycardia and hypokalemia were prognostic factors of both single and acute on chronic theophylline overdose, while hyperglycemia and ingested theophylline dose were prognostic factors of single theophylline overdose. According to the recorded clinical and laboratory parameters all patients were categorized in to mild, moderate and severe. There was significant difference between such classifications as regard pulse rate and systolic blood pressure, serum potassium and random blood glucose levels. Risk factors could not be identified as percentage of mortality rate was not enough.

\section{Recommendations}

1- It is recommended to implement prognostic factors and grading system outlined in this study to evaluate and follow up theophylline intoxicated cases.

2- Perform wide scaled study on patients with acute theophylline intoxication was recommended in order to specify risk factors for those patients.

\section{References}

Borkan S (2002): Extracorporeal therapies for acute intoxications. Critical Care Clinics. Journal; vol.18:393-420.

Caravati E (2004): "Smooth Muscle Relaxants" In: Medical toxicology. Dart R. (eds.). 3rd edition. Lippincott Williams \& Wilkins, Ch.166, pp.1009- 13.

Henry J and Minton N (2011): Treatment of theophylline overdose. American Journal of Emergency Medicine; vol .14:606.

Hoffman R (2006): Methylxanthines and selective B2 adrenergic agonist. In: Goldfrank's toxicological emergencies. Boyle P., Edmonson K. and Wonsiewicz M. (eds.). $8^{\text {th }}$ edition. McGrw-Hill Companies, Inc. Ch.63, pp. 989-1000.

Kato T, Nakano H, and Okada K (2007): A clinical review of patients with convulsions during theophylline administration II: points to consider in administration and prognosis as determined in patients with theophyline toxicosis. Arerugi Journal; vol. 56: 691-8.

Kumar T (2004): Acute Respiratory Infection with CNS Excitation Symptoms-Consider Theophylline
Over- Dosage. Indian Pediatrics Journal; vol. 41:1066-67.

Lim S, Tan S, Tai D et al., (2005): Successful treatment of theophylline toxicity with continuous venovenous haemofiltration. Critical Care and Shock Journal; vol.8: 96 - 7.

Lowry J, Jarrett R, Wasserman G et al., (2001): Theophylline Toxicokinetics in Premature Newborns. Archives of Pediatrics and Adolescent Medicine Journal; vol. 155:934-939.

Perry H (2011): Theophylline poisoning Quoted from http://www.uptodate.com/contents/theophyllinepoisoning.

Rahat K, Baljit S, Narinder S et al., (2005): Review of Management of Common Poisoning in India. Int. Medical Journal; vol.5; 04-06.

Raymond Y (2010): Home " Respiratory Airway Medications: Select Toxicities and Drug Interactions. Quoted from: C:/users/win 7/Documents/Downloads/Discussonseizures2010. mht

Reilly H (2008): eMedicine Specialities >Pediatric: Cardiac diseases and Critical care medicine $>$ Toxicology. Quoted from: http://www.wbmd.com/services professional.html

Rutten J, van den Berg B, van Gelder T et al., (2005): Severe theophylline intoxication: a delay in charcoal haemoperfusion solved by oral activated charcoal. Nephrology Dialysis Transplantation Journal; vol.20: 2868-2869.

Scheindlin S (2007): A new look at the Xanthine Alkaloids. Molecular Interventions Journal; vol. 7: 236- 42.

Shannon M (1993): Predictors of major toxicity after theophylline overdose. Annals of Internal Medicine Journal; vol.119:1161-7.

Shannon M. (1999): Life-Threatening Events after Theophylline Overdose. Archives of Internal Medicine Journal; vol.159:989-994

Shannon M and Perry E (2005): Theophylline and other Methyl Xanthine In: Critical care toxicology: diagnosis and management of the critically poisoned patients. Brent J. (eds.). 1st edition. Elsevier Health Sciences, Ch. 40, pp.458-64.

Tesfaye H, Prusa R, and Doupovcová J (2008): Hypokalemia in a suicide attempt of an adolescent girl. Casopis Lekaru Ceskych Journal; vol.147:333-6 


$$
\text { الملخص العربي }
$$

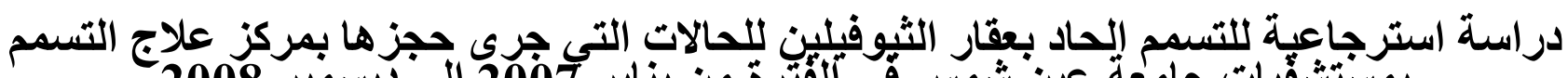

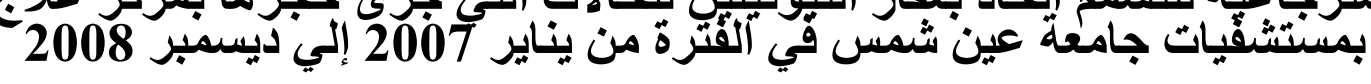

\section{سعد نجيب و أماني السيد و هند الهلالي و سارة سعيد1}

يعتبر الثيوفيلين من موسعات القصبة الهوائية لذلك يستخدم بكثرة في حالات الربو الحاد منذ أكثر من 65 عاما.

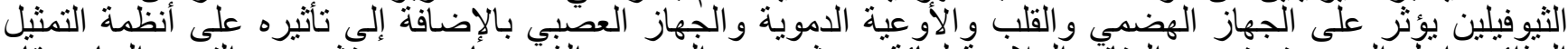

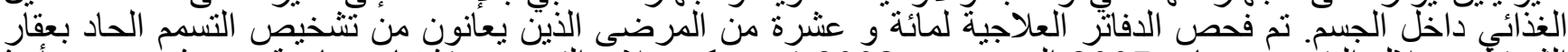

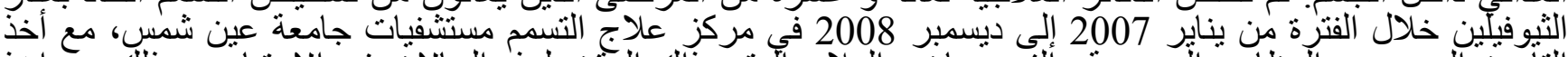

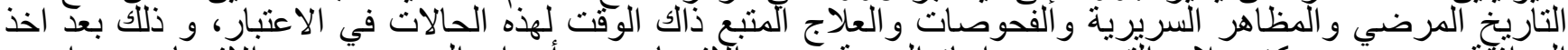

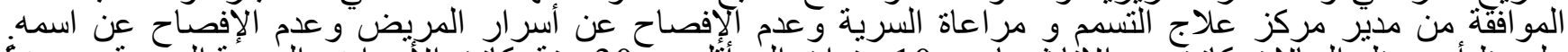

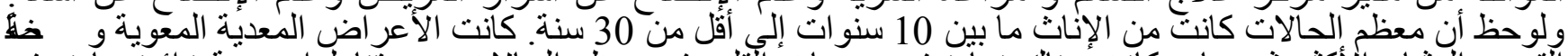

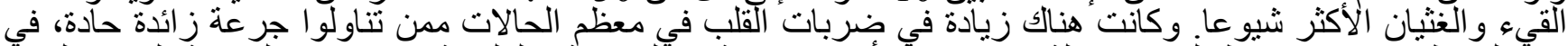

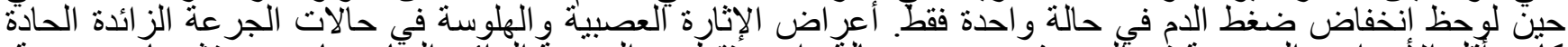

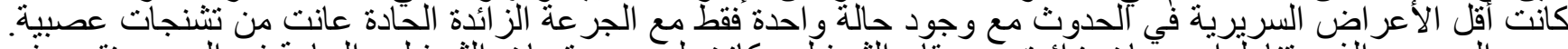

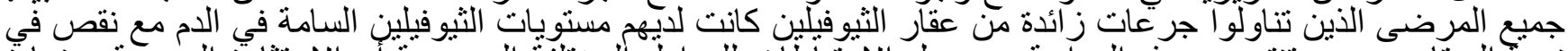

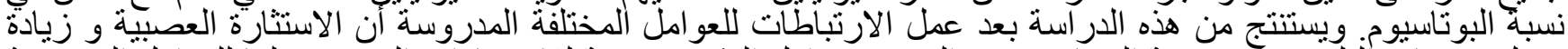

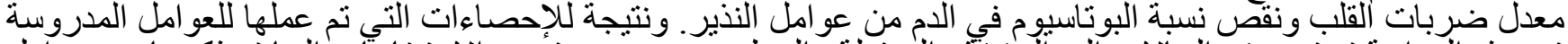

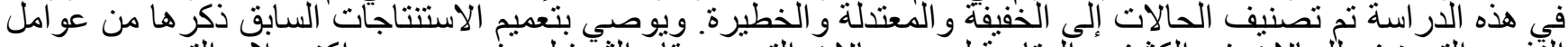

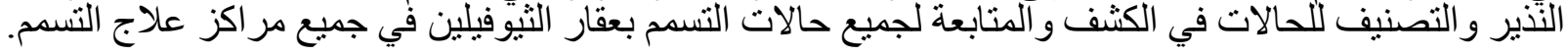

1 قسم الطب الثرعي و السموم الإكلينيكية كلية الطب جامعة عين شمس 